\title{
Originales
}

\section{Síndromes coronarios agudos con elevación simultánea del segmento ST en derivaciones inferiores y precordiales}

\author{
J. ORTEGA CARNICER
}

Servicio de Medicina Intensiva. Hospital General de Ciudad Real. Ciudad Real. España.

Objetivo. Describir los pacientes con dolor torácico y elevación simultánea del segmento ST en las derivaciones inferiores y precordiales derechas.

Material y métodos. Diseño: serie de casos. Ámbito: Servicio de Medicina Intensiva de $16 \mathrm{ca}-$ mas, sin sala de hemodinámica propia, que atiende a pacientes adultos coronarios y polivalentes. Pacientes: se estudiaron 10 pacientes con síndrome coronario agudo que tuvieron: a) una elevación del segmento $S T \geq 1 \mathrm{~mm}$ en dos o más derivaciones contiguas inferiores y precordiales derechas, b) una normalización del segmento ST elevado y/o la aparición de ondas Q o la disminución de la amplitud de la onda $\mathbf{R}$ tras la desaparición del ángor y c) un estudio coronariográfico.

Resultados. Hubo 9 varones y 1 mujer, con una edad media de 62,6 años. Dos pacientes tuvieron historia de infarto de miocardio inferior. Nueve pacientes recibieron tratamiento trombolítico a los 122,2 \pm 93,9 minutos del inicio del ángor. Las derivaciones III y $\mathbf{V}_{3}$ mostraron las mayores elevaciones del segmento ST. Los niveles séricos de creatincinasa fueron normales en 3 casos y significativamente elevados (>1700 U/I) en 6 . EI electrocardiograma evolucionó hacia la normalidad en 2 casos y mostró ondas $Q$ inferiores en 5 pacientes y ondas $T$ negativas en 3 pacientes. Tres pacientes carecieron de complicaciones, 3 casos tuvieron bloqueos cardíacos y otros 3 , taquicardias ventriculares. La arteriografía corona-

Correspondencia: Dr. J. Ortega Carnicer.

C/ Los Alisos, 10

13002 Ciudad Real. España.

Correo electrónico: jortegacar@wanadoo.es

Manuscrito aceptado el 1-XII-2005. ria fue normal en 2 pacientes, 3 tuvieron una lesión proximal en la arteria coronaria derecha y 5 tuvieron dos o más arterias coronarias estenóticas. Tres pacientes fueron diagnosticados de angina inestable, una paciente de miocardiopatía apical transitoria, 4 de infarto agudo de miocardio inferior con afectación ventricular derecha y 2 de infarto agudo de miocardio sin onda $Q$ anterior. Todos los pacientes sobrevivieron.

Conclusiones. La mayoría de los pacientes con síndrome coronario agudo asociado a una elevación simultánea del SST en derivaciones inferiores y precordiales derechas tuvieron una enfermedad coronaria multivaso y todos los pacientes con lesión única de un vaso coronario tuvieron un infarto ventricular derecho secundario a una lesión grave proximal de la arteria coronaria derecha.

PALABRAS CLAVE: síndromes coronarios agudos, infarto agudo de miocardio, angina inestable, elevación simultánea del segmento ST inferior y precordial.

ACUTE CORONARY SYNDROMES WITH SIMULTANEOUS ELEVATION OF THE ST SEGMENT IN INFERIOR AND PRECORDIAL LEADS

Objetive. To describe the patients with ischemic chest pain and simultaneous ST-segment elevation in the inferior and right precordial leads.

Material and methods. Design: series of cases. Context: an adult, Intensive Care Service with 16 beds, with no hemodynamic service, for the management of coronary and polyvalent patients. Patients: we studied 10 patients with acute coronary syndrome who had: a) a ST-segment elevation $\geq 1 \mathrm{~mm}$ in two or more contiguous leads in the inferior and right precordial leads, b) a reso- 
lution of the ST-segment elevation and/or the appearance of $Q$ waves or decrease of the $R$ wave amplitude after the disappearance of the angina and c) a coronary angiographic study.

Results. There were 9 men and 1 woman, with an average age of 62.6 years. Two patients had a background of inferior myocardial infarction. Nine patients received thrombolytic treatment after $122.2 \pm 93.9$ minutes of the pain onset. Leads III and $\mathbf{V}_{3}$ showed the greatest elevations of ST segment. Serum levels of creatine kinase were normal in 3 cases and significantly elevated (>1700 U/L) in six. The ECG evolved to normality in 2 cases and it showed inferior $Q$ waves in 5 patients, and negative $T$ waves in 3 patients. Three patients had no complications, three cases had cardiac blocks and three patients had ventricular tachycardias. The coronary arteriography was normal in 2 patients; three patients showed a proximal stenosis in the right coronary artery and five patients had two or more stenotic coronary arteries. Three patients were diagnosed of unstable angina, one patient was diagnosed of transient apical cardiomyopathy, 4 patients had an acute inferior myocardial infarction with right ventricular extension and 2 patients had a myocardial infarction without anterior $\mathbf{Q}$ wave. All the patients survived.

Conclusions. Most of the patients with acute coronary syndrome associated with simultaneous SST elevation in inferior and right precordial leads had multivessel coronary disease and all patients with only one coronary vessel involved had right ventricular infarction secondary to severe proximal lesion of the right coronary artery.

KEY WORDS: acute coronary syndromes, acute myocardial infarction, unstable angina, simultaneous ST-segment elevation in the inferior and precordial leads.

\section{INTRODUCCIÓN}

El electrocardiograma (ECG) es un medio diagnóstico sencillo y barato que proporciona una buena correlación entre la elevación del segmento ST (SST) y la arteria coronaria responsable de la isquemia miocárdica aguda. En presencia de malestar torácico isquémico, la elevación del SST en las derivaciones precordiales o inferiores suele indicar que existe una obstrucción total (trombótica o vasoespástica) del flujo sanguíneo coronario en la cara anterior o inferior del corazón, respectivamente. Excepcionalmente, el ECG puede mostrar una elevación simultánea del SST en las derivaciones inferiores y anteriores derechas que sugiere la existencia de una enfermedad multivaso que ocasiona una isquemia miocárdica extensa ${ }^{1-12}$. Se presentan 10 pacientes con cardiopatía isquémica que fueron sometidos a un estudio coronariográfico tras presentar angina asociada a elevación simultánea del SST en derivaciones inferiores y precordiales derechas.

\section{PACIENTES Y MÉTODOS}

\section{Pacientes}

El estudio comprende 10 pacientes que fueron ingresados en la Unidad de Cuidados Intensivos (UCI) del Hospital de Alarcos de Ciudad Real entre enero de 1997 y diciembre de 2004. La selección de los enfermos se basó en tres criterios: a) la aparición de malestar torácico isquémico agudo asociado a una elevación del SST $\geq 1 \mathrm{~mm}$ en dos o más derivaciones contiguas inferiores y precordiales derechas $\left(\mathrm{V}_{1}\right.$ a $\mathrm{V}_{3}$ ), b) la normalización del SST elevado y/o la aparición de ondas $\mathrm{Q}$ o disminución de la amplitud de la onda $\mathrm{R}$ tras la desaparición del ángor y c) la realización posterior de un estudio angiográfico coronario.

\section{Electrocardiograma}

Se realizó un ECG estándar $(1 \mathrm{mV}=10 \mathrm{~mm}$, a $25 \mathrm{~mm} / \mathrm{seg}$ ) de 12 derivaciones en todos los pacientes durante el dolor torácico y repetidamente durante la estancia en la UCI. La elevación del SST se midió en milímetros, a $80 \mathrm{mseg}$ del punto J y utilizando el final del segmento PR como línea isoeléctrica. El diagnóstico de infarto agudo de miocardio (IAM) se fundamentó en la presencia de malestar torácico isquémico $>20$ minutos junto a elevación simultánea del SST $\geq 1 \mathrm{~mm}$ en, al menos, dos derivaciones contiguas y posterior elevación de las enzimas cardíacas (creatincinasa $[\mathrm{CPK}]$ y su fracción $\mathrm{MB}$ ) excediendo el percentil 99 de los valores de referencia del grupo control en dos muestras sucesivas o un valor máximo que supera dos veces el límite superior de la normalidad. Con el fin de excluir otras causas de elevación del SST de origen no isquémico (pericarditis agudas, repolarización precoz, hipertrofia ventricular, síndrome de Brugada, etc.), se rechazaron todos los enfermos que no tuvieron normalización del SST con la desaparición del dolor y no mostraron elevación posterior de las enzimas cardíacas. Se consideró que existía una angina inestable cuando desaparecía la elevación del SST con el alivio del ángor y las enzimas cardíacas permanecían dentro de la normalidad. Se definió como angina de Prinzmetal la aparición de malestar torácico en reposo junto a enzimas cardíacas y coronariografía normal. Se determinó el IAM con onda $\mathrm{Q}$ como la aparición de cualquier onda $\mathrm{Q}$ en las derivaciones $\mathrm{V}_{1}$ a $\mathrm{V}_{3}$ o el desarrollo de ondas $\mathrm{Q} \geq 30 \mathrm{mseg}$ en las derivaciones I, II, aVL, aVF, $\mathrm{V}_{4}, \mathrm{~V}_{5}$ o $\mathrm{V}_{6}$, siempre que midieran $1 \mathrm{~mm}$ de profundidad y se visualizaran en dos derivaciones contiguas. Se hizo el diagnóstico de IAM sin onda $\mathrm{Q}$ cuando un ECG registrado > 12 horas del episodio anginoso no mostraba la presencia de ondas $\mathrm{Q}$ con las características descritas antes.

\section{Cateterismo cardíaco}

La angiografía coronaria se realizó usando la técnica de Judkins o, más raramente, de Sones. La ven- 
TABLA 1. Principales datos clínicos y electrocardiográficos

\begin{tabular}{|c|c|c|c|c|c|c|c|c|c|c|c|c|}
\hline Caso & Edad/Sexo & $\begin{array}{l}\text { Antecedentes } \\
\text { personales }\end{array}$ & $\begin{array}{l}\text { Elevación } \\
\text { II III }\end{array}$ & $\begin{array}{l}\mathrm{S}_{\mathrm{S}} \mathrm{ST}(1 \\
\mathrm{F} \mathrm{V}_{1}\end{array}$ & $\begin{aligned}\mathrm{mm}) \\
\mathrm{V}_{2}\end{aligned}$ & $\begin{array}{l}\text { en } d \\
V_{3}\end{array}$ & $\begin{array}{l}\text { leriva } \\
\mathrm{V}_{4}\end{array}$ & $\begin{array}{l}\text { aciones } \\
\mathrm{V}_{5} \mathrm{~V}_{6}\end{array}$ & $\begin{array}{c}\text { Trombolítico/tiempo } \\
(\min )\end{array}$ & $\begin{array}{l}\text { Pico CPK/MB } \\
\text { (U/l) }\end{array}$ & Evolución ECG & $\begin{array}{l}\text { Complicaciones } \\
\text { significativas }\end{array}$ \\
\hline 1 & $70 \mathrm{~V}$ & HTA & 11 & 12 & 3 & 7 & 4 & 31 & $\mathrm{rPA} / 120$ & $2.578 / 107$ & \multirow{5}{*}{$\begin{array}{c}\mathrm{T}(-): \mathrm{V}_{1} \mathrm{a} \mathrm{V}_{5} \\
\mathrm{Q}: \mathrm{II}, \mathrm{III}, \mathrm{aVF} \\
\text { Normal } \\
\mathrm{T}(-) \text { : II y } \mathrm{V}_{2} \mathrm{a} \mathrm{V}_{6} \\
\text { Normal }\end{array}$} & \multirow{5}{*}{$\begin{array}{c}\text { TV } \\
\text { Bloqueo AV completo } \\
- \\
\text { Angina recurrente }\end{array}$} \\
\hline 2 & $74 \mathrm{~V}$ & EPOC. AE & 1010 & 83 & 3 & 5 & 5 & $4-$ & SK/45 & $1.712 / 253$ & & \\
\hline 3 & $48 \mathrm{~V}$ & Fumador & 12 & 21 & 3 & 8 & 6 & $2-$ & $\mathrm{rPA} / 75$ & Normal & & \\
\hline 4 & $74 \mathrm{~V}$ & IM inferior & 11.5 & 11 & 5 & 5 & 5 & 31 & - & Normal & & \\
\hline 5 & $36 \mathrm{~V}$ & Trasplante renal & 0.51 .5 & 14 & 3 & 5 & 4 & $3-$ & $\mathrm{rPA} / 120$ & Normal & & \\
\hline 6 & $65 \mathrm{H}$ & $\begin{array}{c}\text { Diabetes } \\
\text { Estrés psíquico }\end{array}$ & 11 & $1-$ & 2 & 4 & & $1.5-$ & $\mathrm{rPA} / 240$ & $313 / 18$ & \multirow{2}{*}{$\begin{array}{c}\text { T(-): I, II, aVL, } \\
\text { aVF y V } \text { a V }_{6} \\
\text { Q: III, aVF }\end{array}$} & \\
\hline 7 & $67 \mathrm{~V}$ & - & 44 & 41.5 & & 5.5 & & - & $\mathrm{rPA} / 60$ & $1.806 / 206$ & & $\begin{array}{l}\text { BAV Mobitz I. FA } \\
\text { Hipotensión arterial }\end{array}$ \\
\hline 8 & $63 \mathrm{~V}$ & IM inferior. HTA & 11.5 & 11 & 3 & 5 & 8 & 32 & $\mathrm{TNK} / 75$ & $3.515 / 398$ & \multirow{3}{*}{$\begin{array}{l}\text { Q: II, III, aVF; } \\
\text { T(-): } \mathrm{V}_{4} \text { a V } 6 \\
\text { Q: II, III, aVF; } \\
\text { R/S > } 1 \text { en } V_{2} \\
\text { Q: II, III, aVF }\end{array}$} & IVI \\
\hline 9 & $67 \mathrm{~V}$ & Fumador. EPOC & 45 & 42 & 2 & 2 & & -- & $\mathrm{rPA} / 50$ & $2.454 / 320$ & & $\begin{array}{l}\text { TV y DAI. Disfunción } \\
\text { papila mitral }\end{array}$ \\
\hline 10 & $62 \mathrm{~V}$ & Fumador & 13 & 15 & 3 & 1 & & -- & TNK/315 & $2.504 / 283$ & & $\begin{array}{c}\text { TV } \\
\text { Bloqueo } \\
\text { sinoauricular 2. }\end{array}$ \\
\hline
\end{tabular}

HTA: hipertensión arterial; EPOC: enfermedad pulmonar obstructiva crónica; AE: angina de esfuerzo; IM: infarto de miocardio; rPA: reteplasa; SK: estreptocinasa; TNK: tenecteplasa; CPK: creatinfosfocinasa; T(-): onda T negativa; TV: taquicardia ventricular; AV: auriculoventricular; DAI: desfibrilador automático implantable.

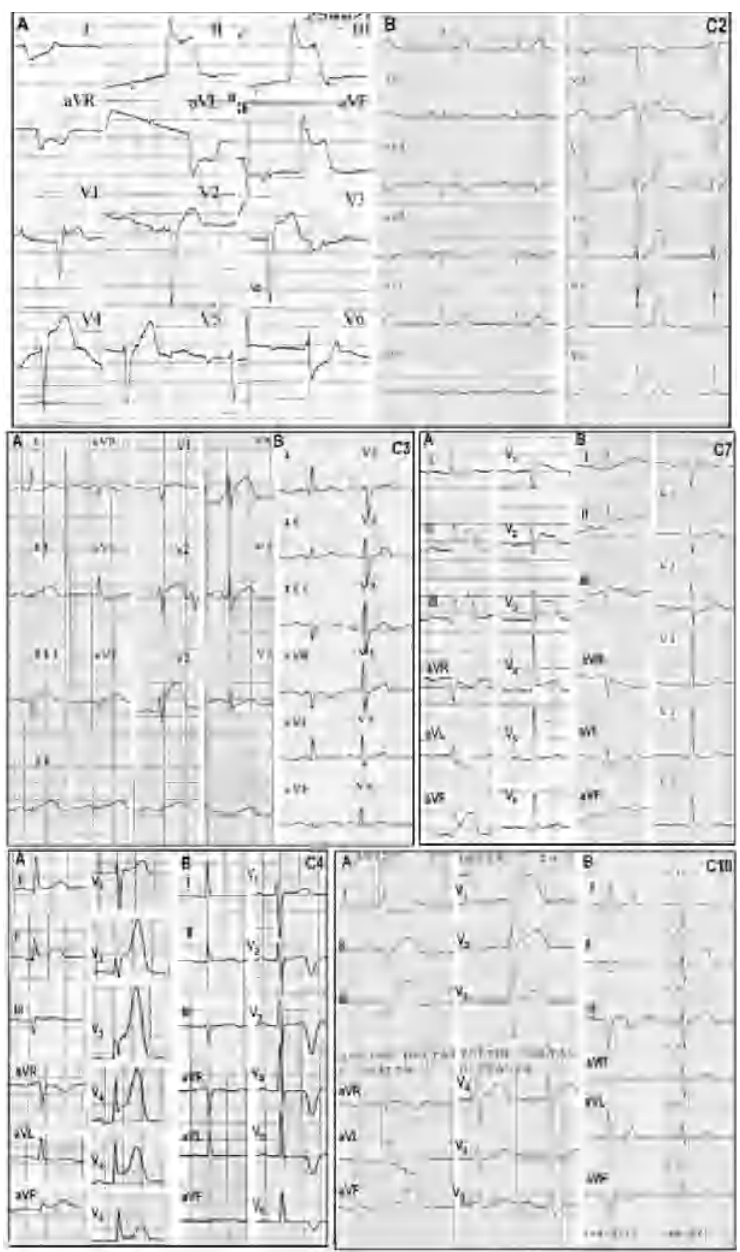

Figura 1. Selección de electrocardiogramas de 12 derivaciones correspondientes a varios casos clínicos (2, 3, 4, 7 y 10) mostrando una elevación simultánea del segmento ST en las derivaciones precordiales derechas e inferiores (A) y su evolución posterior cuando el paciente estaba asintomático tras el tratamiento $(B)$. triculografía izquierda se valoró utilizando la proyección oblicua anterior derecha. La enfermedad arterial coronaria se definió por la presencia de estenosis $\geq$ $50 \%$ en una arteria epicárdica mayor. Cuando en la arteriografía se encontraron $\geq 2$ arterias coronarias mayores afectas, el vaso responsable del síndrome coronario agudo y, por ende, el diagnóstico definitivo se determinó teniendo en cuenta la existencia o ausencia de infarto de miocardio previo, los cambios electrocardiográficos tras la normalización del SST, la presencia de trombo intraluminal en la angiografía y los hallazgos hemodinámicos, ecocardiográficos, ventriculográficos e isotópicos miocárdicos.

\section{RESULTADOS}

Hubo 9 varones y 1 mujer, con una edad media de 62,6 (límites: 36 y 74) años. Las principales características clínicas y electrocardiográficas se sintetizan en la tabla 1. Dos pacientes tenían antecedentes de infarto de miocardio inferior. Excepto el caso 4, todos los enfermos tuvieron más de 20 minutos de malestar torácico isquémico y recibieron tratamiento trombolítico a los 122,2 \pm 93,9 minutos del inicio del ángor. Las derivaciones electrocardiográficas III y $\mathrm{V}_{3}$ mostraron las mayores elevaciones del SST (fig. 1). Los niveles séricos de CPK estuvieron dentro de la normalidad en tres casos, ligeramente elevados (300 U/l) en un caso y significativamente elevados (> $1700 \mathrm{U} / \mathrm{l})$ en seis casos.

El ECG evolucionó hacia la normalidad en 2 casos, mostró ondas Q inferiores en 5 casos (uno de ellos tuvo además ondas T negativas anteriores), ondas $\mathrm{T}$ negativas en derivaciones anteriores en 2 casos y ondas $\mathrm{T}$ negativas difusas en un caso.

Tres pacientes no presentaron complicaciones clínicas, otros tres tuvieron bloqueos cardíacos, tres casos más sufrieron taquicardias ventriculares y uno de ellos requirió el implante de un desfibrilador automático implantable. 
TABLA 2. Principales datos angiográficos y diagnóstico definitivo

\begin{tabular}{|c|c|c|c|c|c|c|c|}
\hline \multirow{2}{*}{ Caso } & \multicolumn{2}{|r|}{ Ventriculografía izquierda } & \multicolumn{3}{|c|}{ Angiografía coronaria (\% diámetro obstrucción) } & \multirow{2}{*}{ Intervención coronaria } & \multirow{2}{*}{ Diagnóstico definitivo } \\
\hline & $\mathrm{FE}(\%)$ & Movimiento regional pared & DA & $\mathrm{CX}$ & $\mathrm{CD}$ & & \\
\hline 1 & 56 & Acinesia apical & $90 \%$ media & $\begin{array}{l}80 \% \text { proximal } \\
75 \% \text { distal }\end{array}$ & $\begin{array}{l}60 \% \text { distal } \\
90 \% 1 .^{\mathrm{a}} \mathrm{OM}\end{array}$ & $\mathrm{ACTP}+$ Stent DA y CX & IAMNQ anterior \\
\hline 2 & 65 & $\begin{array}{l}\text { Hipocinesia posterobasal } \\
\text { Hipocinesia diafragmática }\end{array}$ & Normal & Normal & $90 \%$ proximal & No revascularizable & IAM inferior + AVD \\
\hline 3 & 70 & Normal & Normal & Normal & (D) Normal & No & AP anterior e inferior \\
\hline 4 & 67 & Hipocinesia posterobasal & $90 \%$ media & Normal & $\begin{array}{l}\text { (D) } 90 \% \text { proximal } \\
75 \% \text { media y cruz }\end{array}$ & ACTP + Stent DA proximal & $\mathrm{AI}$ anterior \\
\hline 5 & 62 & Normal & $90 \%$ distal & $70 \%$ bisectriz & (D) $90 \%$ rama VD & No revascularizable & AI anterior e inferior \\
\hline 6 & 62 & $\begin{array}{l}\text { Discinesia apical } \\
\text { transitoria }\end{array}$ & $50 \% 1 .^{a} \mathrm{D}$ & Normal & (D) Normal & No & Miocardiopatía apical \\
\hline 7 & 45 & Acinesia inferior & Normal & Normal & (D) $90 \%$ proximal & ACTP + Stent CD & IAM inferior + AVD \\
\hline 8 & 35 & $\begin{array}{l}\text { Acinesia inferior } \\
\text { Hipoquinesia anterior }\end{array}$ & $75 \%$ media & (D) $60 \%$ media & $75 \%$ media & $\mathrm{ACTP}+$ Stent DA y CD & IAMNQ anterior \\
\hline 9 & 40 & $\begin{array}{l}\text { Acinesia inferior, } \\
\text { posterior y lateral }\end{array}$ & $90 \%$ proximal & $80 \%$ media & (D) $95 \%$ proximal & Cirugía & IAM inferior + AVD \\
\hline 10 & 55 & Hipocinesia posterior & Normal & Normal & (D) $98 \%$ proximal & $\mathrm{ACTP}+$ Stent CD & IAM inferior + AVD \\
\hline
\end{tabular}

FE: fracción de eyección; DA: arteria coronaria descendente anterior; CX: arteria coronaria circunfleja; CD: arteria coronaria derecha; (D): dominante; OM: arteria coronaria oblicu marginal; D: arteria coronaria diagonal; ACTP: angioplastia coronaria percutánea; IAM: infarto agudo de miocardio; IAMNQ: infarto agudo de miocardio sin onda Q; AP: angina de marginal; D: arteria coronaria diagonal; ACTP: angioplastia coronaria

Los resultados de la arteriografía coronaria se muestran en la tabla 2. Los estudios hemodinámicos se llevaron a cabo a los $8 \pm 5,6$ días del ingreso. Ningún paciente tuvo enfermedad del tronco común de la arteria coronaria izquierda. Siete pacientes tuvieron, al menos, una arteria coronaria epicárdica mayor con una lesión crítica $\geq 90 \%$. Dos casos tuvieron arterias coronarias normales; tres pacientes tuvieron sólo un vaso afecto y cinco casos mostraron lesiones en dos o más arterias coronarias. Se realizó una intervención coronaria percutánea en cuatro pacientes, dos de ellas sobre la arteria coronaria descendente anterior izquierda.

Tres pacientes fueron diagnosticados de angina inestable, uno de ellos de tipo vasoespástico (Prinzmetal) con arterias coronarias normales. La única mujer incluida en el estudio fue diagnosticada de miocardiopatía apical transitoria (miocardiopatía takotsubo) en base al sexo, la presencia de estrés psíquico intenso (durante el entierro de un familiar) como desencadenante del cuadro, la elevación del SST, la escasa elevación enzimática, la presencia de arterias coronarias normales y la dilatación apical transitoria detectada en la ecocardiografía y la ventriculografía. Cuatro pacientes fueron diagnosticados de IAM inferior con afectación ventricular derecha. Todos los pacientes sobrevivieron y fueron dados de alta del hospital.

\section{DISCUSIÓN}

Aunque la presencia de malestar torácico isquémico asociado a una elevación simultánea del SST en las derivaciones inferiores y precordiales derechas sugiere que existe una obstrucción arterial coronaria doble que explica la isquemia miocárdica aguda de la cara inferior y anterior del corazón, estos hallazgos electrocardiográficos pueden producirse por la obstrucción aguda de un único vaso arterial coronario. Así, a tenor de los datos presentados y la bibliografía existente ${ }^{1-12}$, las obstrucciones coronarias agudas que pueden dar lugar a una elevación simultánea del SST en la cara inferior y anterior del corazón son las siguientes:

1) Obstrucción proximal de una arteria coronaria derecha dominante: algunos pacientes con IAM inferior muestran en el ECG una corriente de lesión simultánea en las derivaciones inferiores y anteriores (hasta $\mathrm{V}_{5}$ ). La evolución electrocardiográfica posterior muestra la aparición de ondas Q en la cara inferior y la normalización del ECG (resolución del SST y ausencia de ondas Q) en las derivaciones precordiales. Este patrón electrocardiográfico de IAM inferior se acompaña también de datos hemodinámicos, ecocardiográficos e isotópicos de infarto ventricular derecho. El estudio angiográfico coronario revela una estenosis de la arteria coronaria derecha y un árbol coronario izquierdo normal ${ }^{1-3,5,6,8}$. Aunque la mayoría de las obstrucciones de la arteria coronaria derecha suelen situarse en la porción proximal, a veces la obstrucción de una rama ventricular derecha (marginal) que irriga el ventrículo derecho puede producir idénticos cambios electrocardiográficos ${ }^{1,3,8}$. Ocasionalmente, la obstrucción de esta rama ventricular derecha puede causar un infarto aislado del ventrículo derecho que se manifiesta en el ECG con una elevación del SST limitada a las derivaciones precordiales derechas, lo que entraña una mayor dificultad diagnóstica, ya que el SST en la cara inferior está isoeléctrico ${ }^{7,11}$ o descendido ${ }^{1,4}$.

Las inusuales elevaciones del SST en las derivaciones precordiales debidas a la oclusión de la arteria coronaria derecha suelen decrecer desde la derecha a la izquierda (caso 10, fig. 1) y luego no aparecen ondas $\mathrm{Q}$ (casos 7 y 10, fig. 1), mientras que cuando corresponden a un típico infarto anterior del ventrículo izquierdo la elevación del SST usualmente aumenta desde la derecha a la izquierda y 
después aparecen ondas Q anteriores. Además, la elevación del SST anterior secundario a un infarto del ventrículo derecho suele mostrar un aspecto abovedado $(2,5-7,11)$ y no suele sobrepasar la derivación $\mathrm{V}_{4}$, salvo en dos de los cinco casos descritos por Geft et $\mathrm{al}^{1}$.

En la mayoría de los pacientes con infarto del ventrículo derecho no es posible visualizar esta elevación del SST en la cara anterior por las fuerzas dominantes correspondientes a la lesión de la pared inferior del ventrículo izquierdo. Estos cambios electrocardiográficos podrían explicarse por una dilatación progresiva del ventrículo derecho y el desarrollo de una rotación horaria, lo cual ocasionaría que las derivaciones $\mathrm{V}_{1} \mathrm{a} \mathrm{V}_{4-5}$ registraran los fenómenos eléctricos del ventrículo derecho como sucede a veces en el embolismo pulmonar agudo $^{13}$

2) Obstrucción de una arteria coronaria descendente anterior izquierda larga y distal al origen de su primera diagonal: los pacientes con IAM anterior debido a la obstrucción de una arteria coronaria descendente anterior izquierda larga (que rodea la punta del corazón e irriga parte de la pared inferior del ventrículo izquierdo) y distal al origen de su primera rama diagonal pueden tener también una elevación simultánea del SST en las derivaciones anteriores e inferiores ${ }^{14-16}$. Estos pacientes, a diferencia de los IAM anteriores que se acompañan de un SST normal o descendido en la cara inferior debido a una obstrucción proximal de la arteria descendente anterior izquierda, suelen tener un buen pronóstico hospitalario, pues la obstrucción distal de la arteria descendente anterior izquierda ocasiona que la masa miocárdica necrótica sea más pequeña ${ }^{12,17}$. Así, Yip et $\mathrm{al}^{17}$ estudiaron retrospectivamente 924 pacientes a los que realizaron una intervención coronaria percutánea por IAM y encontraron 37 pacientes (4\%) que tenían una elevación del SST $\geq 1 \mathrm{~mm}$ en las derivaciones inferiores y precordiales. Ocho de estos 37 pacientes, con una obstrucción de una arteria coronaria descendente anterior izquierda larga, mostraron una elevación del SST que sumaba $<3 \mathrm{~mm}$ en las derivaciones inferiores, una enfermedad coronaria limitada a un vaso y un pronóstico favorable $(0 \%$ de mortalidad a los 30 días). En cambio, los 29 enfermos restantes que no tuvieron este tipo de lesión coronaria tuvieron una elevación del SST inferior que sumaba $>11 \mathrm{~mm}$, una afectación coronaria multivaso y más complicaciones clínicas que elevaban la mortalidad (48,3\% a los 30 días).

3) Obstrucción de una pequeña arteria coronaria derecha que irriga predominantemente el ventrículo derecho cuando la pared inferior del ventrículo izquierdo recibe el flujo sanguíneo de una arteria coronaria circunfleja izquierda dominante.

4) Obstrucción de una arteria coronaria descendente anterior izquierda que proporciona flujo colateral a la arteria coronaria derecha previamente obstruida o, su imagen en espejo, obstrucción de una arteria coronaria derecha que aporta flujo a la arteria coronaria descendente anterior izquierda ocluida
5) Arterias coronarias normales con vasoespasmo sobreañadido: Misumi et $\mathrm{al}^{18}$ refirieron un paciente con dolor torácico asociado a elevación del SST anterior e inferior durante la realización de una arteriografía coronaria que mostró, por primera vez en la literatura, la aparición de un espasmo espontáneo simultáneo en las arterias coronarias descendente anterior izquierda y derecha. El caso 3 podría justificarse por un mecanismo similar, pero también cabría la posibilidad de un espasmo aislado sobre cualquiera de las dos arterias coronarias que actuase por un mecanismo parecido a los comentados con anterioridad.

En 1986, Janosik et $\mathrm{al}^{9}$ describieron el caso de una mujer joven que tuvo dolor torácico prolongado y una elevación simultánea del SST en las derivaciones inferiores y anteriores. Tras administrar morfina y nitroglicerina, la elevación del SST regresó a la línea isoeléctrica, aparecieron ondas Q en ambas caras cardíacas y el valor máximo de la CPK llegó a $400 \mathrm{U} / \mathrm{l}$. La angiografía coronaria fue normal y la ventriculografía izquierda demostró una acinesia apical. Los autores achacaron la elevación del SST a un vasoespasmo simultáneo y mantenido de la arteria coronaria derecha y descendente anterior izquierda. El caso 6 tuvo rasgos comunes al descrito por Janosik et $\mathrm{al}^{9}$ como la elevación del SST anterior e inferior, la escasa elevación de la CPK (300 U/1), las arterias coronarias normales y la ventriculografía con acinesia apical; mientras que difería en cuanto que era una mujer mayor con estrés psíquico como precipitante del cuadro anginoso y que fue tratada con un fibrinolítico. Ambos casos poseen características de la miocardiopatía apical transitoria (miocardiopatía takotsubo), un nuevo síndrome descrito por autores japoneses ${ }^{19,20}$, que tiene afectación predominante por el sexo femenino, especialmente mayores de 60 años, estrés psíquico como factor desencadenante, síntomas similares al IAM, escasa elevación enzimática, arterias coronarias normales y ventriculografía o ecocardiografía con aquinesia apical (transitoria) e hipercinesia basal ${ }^{19,20}$. Aunque en la miocardiopatía apical transitoria no se han descrito elevaciones simultáneas del SST inferior y anterior, la fisiopatología de la miocardiopatía es una gran liberación de catecolaminas que origina un vasoespasmo en las arterias coronarias epicárdicas o, más probablemente, a nivel microvascular.

En conclusión, la mayoría de los pacientes con síndrome coronario agudo asociado a una elevación simultánea del SST en derivaciones inferiores y precordiales derechas tuvieron una enfermedad coronaria multivaso y todos los pacientes con lesión única de un vaso tuvieron un infarto ventricular derecho secundario a una lesión grave proximal de la arteria coronaria derecha.

\section{Declaración de conflicto de intereses}

Los autores han declarado no tener ningún conflicto de intereses 


\section{BIBLIOGRAFÍA}

1. Geft IL, Shah PK, Rodríguez L, Hulse S, Maddahi J, Berman DS, et al. ST elevations in leads $V_{1}$ to $V_{5}$ may be caused by right coronary artery occlusion and acute right ventricular infarction. Am J Cardiol. 1984;53:991-6.

2. Halkett JA, Commeford PJ, Millar RS. Right ventricular infarction mimicking extensive anterior infarction. Chest. 1986;90: 617-9.

3. Bellamy GR, Hollman J. Isolated right ventricular infarction following percutaneous transluminal coronary angioplasty. Am Heart J. 1986;111:168-9.

4. Marchena EJ, Palomo AR, Trohman RG, Myerburg RJ, Kessler KM. Angiographically demonstrated isolated acute right ventricular infarction presenting as $\mathrm{ST}$ elevation in leads $\mathrm{V}_{1}$ to V. Am Heart J. 1987;113:391-3.

5. Kataoka H, Kanzaki K, Mikuriya Y. Massive ST-segment elevation in precordial and inferior leads in right ventricular myocardial infarction. J Electrocardiol. 1988;21:115-20

6. Ilia R, Goldfarb B, Ovsyshcher IA. Concomittant ST elevation in inferior and anterior leads in acute myocardial infarction. J Electrocardiol. 1990;23:199-205.

7. Grollier G, Scanu P, Gofard M, Lognoné T, Valette B, Bureau G, et al. Sus-décalage du segment ST dans les dérivations précordiales antérieures et infarctus du ventricule droit. Arch Mal Coeur. 1992;85;67-75.

8. Fernández AR, de Marchena EJ, Sequeria RF, Kessler KM. Acute right ventricular infarction mimicking extensive anterolateral wall injury. Chest. 1993;104:965-7.

9. Janosik DL, Labovitz AJ, Kennedy HL. Anterior and inferior myocardial infarction in a young woman with angiographically normal coronary arteries. Am Heart J. 1986;112;606-9.

10. Lew AS, Hod H, Cercek B, Shah PK, Ganz W. Inferior ST segment changes during acute anterior myocardial infarction: A marker of the presence or absence of concomitant inferior wall ischemia. J Am Coll Cardiol. 1987;10:519-26.

11. Logeart D, Himbert D, Cohen-Solal A. ST-segment elevation in precordial leads. Anterior or right ventricular myocardial infarction? Chest. 2001;119:290-2.
12. Sadanandan S, Hochman JS, Kolodziej A, Criger DA, Ross A, Selvester R, et al. Clinical and angiographic characteristics of patients with combined anterior and inferior ST-segment elevation on the initial electrocardiogram during acute myocardial infarction. Am Heart J. 2003;146:653-61.

13. Cutforth RH, Oram S. The electrocardiogram in pulmonary embolism. Br Heart J. 1958;20:41-60.

14. Sapin PM, Musselman DR, Dehmer GJ, Cascio W Implications of inferior ST-segment elevation accompanying anterior wall acute myocardial infarction for the angiographic morphology of the left anterior descending coronary artery morphology and site of occlusion. Am J Cardiol. 1992;69:860-5.

15. Tamura A, Kataoka H, Nagase K, Mikuriya Y, Nasu M. Clinical significance of inferior ST elevation during acute anterior myocardial infarction. Br Heart J. 1995;74:611-4.

16. Sasaki K, Yotsukura M, Sakata K, Yoshino H, Ishikawa $\mathrm{K}$. Relation of ST-segment changes in inferior leads during anterior wall acute myocardial infarction to length and occlusion site of the left anterior descending coronary artery. Am J Cardiol. 2001;87:1340-5.

17. Yip HK, Chen MC, Wu CJ, Chang HW, Yu TH, Yeh KH, et al. Acute myocardial infarction with simultaneous ST-segment elevation in the precordial and inferior leads. Chest. 2003;123: 1170-89.

18. Misumi I, Tsuruta $K$, Tanae $H$, Sakamoto $T$, Tsunoda $R$, Sakano S, et al. Successful documentation by coronary angiography of spontaneous simultaneous multivessel coronary spasm in a variant angina patient: a case report. Kokyu To Junkan. 1993;41:1101-5.

19. Tsuchihashi $K$, Ueshima $K$, Uchida $T$, Oh-mura $N$, Kimura K, Owa M, et al. Transient left ventricular apical ballooning without coronary artery stenosis: A novel heart syndrome mimicking acute myocardial infarction. J Am Coll Cardiol. 2001; 38:11-8.

20. Kirisu S, Sato H, Kawagoe T, Ishihara M, Shimatani Y, Nishioka K, et al. Takotsubo-like left ventricular dysfunction with ST-segment elevation: A novel cardiac syndrome mimicking acute myocardial infarction. Am Heart J. 2002;143:448-55. 